\title{
Optimizing human in vivo dosing and delivery of $\beta$-alanine supplements for muscle carnosine synthesis
}

\author{
Trent Stellingwerff • Jacques Decombaz • \\ Roger C. Harris $\cdot$ Chris Boesch
}

Received: 22 October 2011 / Accepted: 2 February 2012/Published online: 23 February 2012

(C) Springer-Verlag 2012

\begin{abstract}
Interest into the effects of carnosine on cellular metabolism is rapidly expanding. The first study to demonstrate in humans that chronic $\beta$-alanine (BA) supplementation $(\sim 3-6 \mathrm{~g}$ BA/day for $\sim 4$ weeks) can result in significantly augmented muscle carnosine concentrations $(>50 \%)$ was only recently published. BA supplementation is potentially poised for application beyond the niche exercise and performance-enhancement field and into other more clinical populations. When examining all BA supplementation studies that directly measure muscle carnosine $(n=8)$, there is a significant linear correlation between total grams of BA consumed (of daily intake ranges of 1.6-6.4 g BA/day) versus both the relative and absolute increases in muscle carnosine. Supporting this, a recent dose-response study demonstrated a large linear dependency $\left(R^{2}=0.921\right)$ based on the total grams of BA consumed over 8 weeks. The pre-supplementation baseline carnosine or individual subjects' body weight (from 65 to $90 \mathrm{~kg}$ ) does not appear to impact on subsequent carnosine synthesis from BA consumption. Once muscle carnosine is augmented, the washout is very slow ( $\sim 2 \% /$ week). Recently, a slow-release BA tablet supplement has been
\end{abstract}

T. Stellingwerff $\cdot$ J. Decombaz

Nestlé Research Center, Lausanne, Switzerland

T. Stellingwerff $(\bowtie)$

Canadian Sports Center: Pacific, Pacific Institute

for Sport Excellence, 4371 Interurban Road,

Victoria, BC V9E 2C5, Canada

e-mail: tstellingwerff@cscpacific.ca

R. C. Harris

Junipa Ltd, Newmarket, UK

C. Boesch

University of Bern, Bern, Switzerland developed showing a smaller peak plasma BA concentration and delayed time to peak, with no difference in the area under the curve compared to pure BA in solution. Further, this slow-release profile resulted in a reduced urinary BA loss and improved retention, while at the same time, eliciting minimal paraesthesia symptoms. However, our complete understanding of optimizing in vivo delivery and dosing of BA is still in its infancy. Thus, this review will clarify our current knowledge of BA supplementation to augment muscle carnosine as well as highlight future research questions on the regulatory points of control for muscle carnosine synthesis.

Keywords $\beta$-Alanine $\cdot$ Carnosine $\cdot$ Muscle $\cdot$ Synthesis . Washout · Dose-response

\section{Introduction}

Carnosine was first identified in beef muscle extract over 100 years ago by the Russian biochemist Gulewitsch (Gulewitsch and Amiradzibi 1900). Given its high concentration in muscle it was suitably named, as carno translated from Latin means "flesh/meat". The relatively high concentration of carnosine in all vertebrate skeletal muscle suggested that it likely serves a physiologically imperative role and mounting evidence, especially over the last several years, has provided more evidences for this. Carnosine ( $\beta$-alanyl-L-histidine) is made up of the proteinogenic essential amino acid L-histidine and the naturally occurring non-essential amino acid $\beta$-alanine (BA; Fig. 1). The concentrations of histidine-containing dipeptides (HCD; e.g. carnosine, anserine, balenine) were first quantified in human skeletal muscle in the mid-1970s (Bergstrom et al. 1974), with carnosine being the only 
Fig. 1 Schematic of the points of regulation for carnosine/ $\beta$-alanine delivery and subsequent skeletal muscle carnosine synthesis

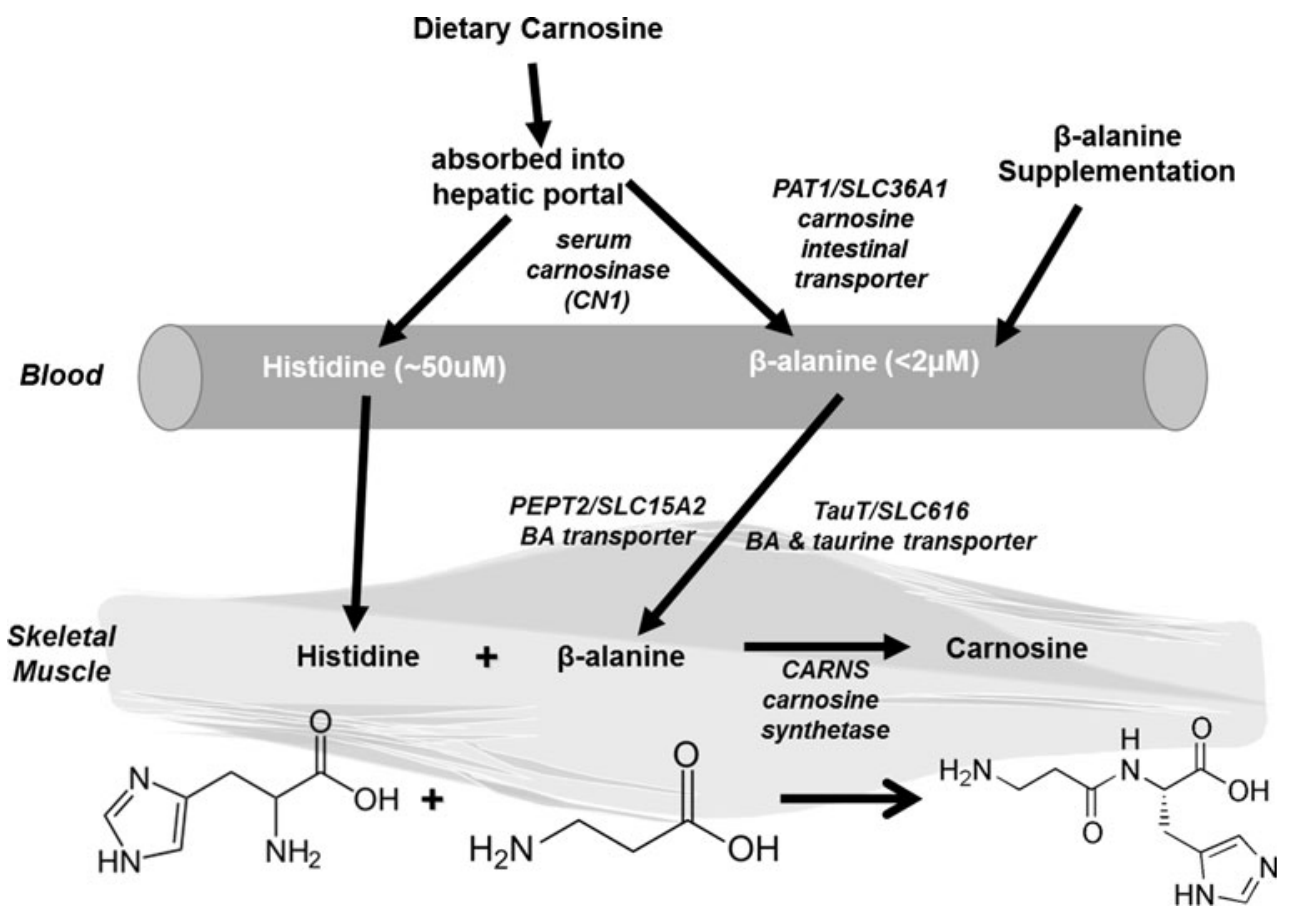

HCD in human skeletal muscle (Quinn et al. 1992), but some sixfold lower than the carnosine content of thoroughbred race horses (Harris et al. 1990). Intra-human and interspecies comparisons have shown strong positive correlations between muscle buffering capacity, carnosine/ HCD levels and fast-twitch muscle percentage (Abe 2000; Harris et al. 1990; Parkhouse et al. 1985). Already since the mid-1980s, it has been known that sprinters and rowers, as compared to marathon runners, have a significantly greater percentage of Type II fast-twitch fibers, and accordingly, greater muscle carnosine concentrations, muscle buffering capacity and high-intensity exercise performance (Parkhouse et al. 1985). Lastly since 2006, eight different studies have clearly shown that prolonged BA supplementation (1.6-6.4 g/day for $>4$ weeks) in humans results in a 40-65\% increase in muscle carnosine content (Kendrick et al. 2008, 2009; Harris et al. 2006; Hill et al. 2007); Baguet et al. 2009, 2010; Derave et al. 2007; Stellingwerff et al. 2011). Thus in recent years, our knowledge on BAsupplemented muscle carnosine increases has developed rapidly.

As evidenced by the diverse topical list of this journals special edition (Derave and Sale 2011), the interest in the effects of carnosine metabolism on prospective functions in myocellular homeostasis and other clinical populations with muscle myopathies or dysfunction are quickly expanding. As extensively reviewed by Begum et al. (2005), primarily, in vitro and pre-clinical research has suggested a multitude of physiological roles for carnosine, including acting as an antioxidant, a regulator of muscle excitation-contraction coupling via increasing calcium sensitivity, a source of histidine for histamine synthesis for wound healing, protecting proteins against glycation during aging. Given the unquestionable role that muscle carnosine plays in augmenting intramuscular buffering (Abe 2000), recent in vivo work has shown strong associations between increased muscle carnosine and high-intensity exercise performance (for reviews see: Sale et al. 2010; Derave et al. 2010; Artioli et al. 2010). Further, as highlighted within this special issue, the efficacy of carnosine and/or BA supplementation to augment muscle carnosine has also been linked to clinical populations, such as Type II diabetics (Gualano et al. 2011) and the elderly (del Favero et al. 2011). Similar to creatine supplementation in humans which has demonstrated efficacy in a range of clinical and disease state populations (Tarnopolsky 2011), BA supplementation leading to augmented muscle carnosine concentrations is potentially poised for applications beyond the niche role of intramuscular buffering to enhance high-intensity exercise performance. Thus, having a sound understanding of the optimal in vivo delivery and dosing of BA supplements to augment muscle carnosine is necessary. Correspondingly, this review will highlight our current knowledge on muscle specific dose-responses; whether baseline levels of muscle carnosine impact subsequent increases; examine the postBA supplementation washout of carnosine; as well as identify future research questions based on the points of regulation of carnosine synthesis. Taken together, this review hopes to elucidate and clarify the BA prescriptive application to augment muscle carnosine. 


\section{Points of regulation for $\beta$-alanine delivery and carnosine synthesis}

Given that the first human study to successfully augment muscle carnosine via BA supplementation only occurred in 2006 (Harris et al. 2006), and given the large species differences in carnosine metabolism (Abe 2000), our understanding of all the points of regulation for BA delivery and carnosine synthesis in humans is only starting to emerge. As with any amino acid/protein, a given content is the result of the equilibrium between synthesis and degradation/elimination. Thus, carnosine loading results in situations where adequate substrates (BA and L-histidine) result in greater carnosine-synthesis rates as compared to degradation rates. Conversely, carnosine washout will result when degradation/elimination exceeds the synthesis (see "Muscle carnosine washout"). Although there are many tissues rich in carnosine, including kidney, olfactory tissue in the brain and reproductive organs (Boldyrev and Severin 1990), this review will primarily focus on skeletal muscle carnosine. Muscle carnosine synthesis appears to be primarily driven by sarcoplasmic BA availability, and thus the three main regulatory steps to consider include: (1) plasma BA delivery, (2) BA transport into the muscle, and (3) the activity of muscle carnosine synthase (CARNS).

Humans have evolved processes to synthesis endogenous BA in the liver from the degradation of uracil (Fritzson 1957), but given that vegetarians have lower muscle carnosine contents (Everaert et al. 2011) optimal dietary delivery of BA (primarily meat or supplementation) is required for significant plasma BA delivery for muscle carnosine synthesis. BA transport across the gut is accomplished by the $\mathrm{H}^{+}$-coupled amino-acid transporter PAT1/ SLC36A1, which is also known as the imino-acid carrier (Thwaites and Anderson 2007). In humans, it appears that BA is readily transported across the gastrointestinal tract (Harris et al. 2006), and thus, this step does not appear to limit muscle carnosine synthesis. Contrary to rodents (Sauerhofer et al. 2007), humans have a highly active serum carnosinase (CN1; Teufel et al. 2003), as the intact carnosine dipeptide is rapidly hydrolyzed to its constituent amino acids with complete hydrolysis within $\sim 3 \mathrm{~h}$ (Gardner et al. 1991). A recent paper has also demonstrated that females have a higher CN1 activity compared to males (Everaert et al. 2011). It could be hypothesized that a lower serum $\mathrm{CN} 1$ activity, as found in males, might cause a longer period of elevated circulating plasma carnosine resulting in increased muscle carnosine transport and synthesis, which is supported by the fact that two recent papers have found higher muscle carnosine contents in males than females (Everaert et al. 2011; Baguet et al. 2011a).

Following plasma carnosine hydrolysis by $\mathrm{CN} 1$ to L-histidine and BA, or direct BA supplementation, BA can then be transported to target tissues. L-Histidine in muscle and plasma is high compared to the low concentrations of $\mathrm{BA}$, and transport of $\mathrm{BA}$ into the muscle cell is via a $\beta$-amino-acid transporter that is strongly dependent on sodium and chloride and has a BA transporter $K_{\mathrm{m}}$ of $\sim 40 \mu \mathrm{M}$ (Bakardjiev and Bauer 1994). However, these transport data were generated via embryonic chicken-muscle cell cultures, and thus, caution should be taken when extrapolating to humans, as this data should be confirmed using human myocyte preparations. In humans in normal physiological situations, the concentration of plasma BA is below the muscle transport $K_{\mathrm{m}}$ of $40 \mu \mathrm{M}$ (Decombaz et al. 2011). Therefore, it could be hypothesized that sarcoplasmic BA delivery is the rate-limiting factor for muscle carnosine synthesis via CARNS (Fig. 1). Both peptide transporter 2 (PEPT2/SLC15A2; Lu and Klaassen 2006; Dieck et al. 1999) and the taurine transporter (TauT/SLC616; Tomi et al. 2008), have been shown to transport BA in several rodent and cell-culture preparations, but remain to be clearly identified as the primary BA transporter in human skeletal muscle. Correspondingly, a recent conference abstract showed in humans that BA supplementation-induced increases in muscle carnosine resulted in the opposite and compensatory changes in muscle taurine content (Harris 2010). This finding presumably was a result of competitive inhibition of taurine transport by BA, lending support to key role that the taurine transporter (TauT) plays in muscle carnosine and taurine contents in humans.

Once BA is transported to the muscle sarcolemma, carnosine synthesis results from BA and ample sarcoplasmic L-histidine by CARNS. Recently, Drozak et al. (2010), using recombinant human protein, have cloned and identified human CARNS, showing that L-histidine exhibits an enzyme $K_{\mathrm{m}}(\sim 37 \mu \mathrm{M})$ four-times greater than BA. However, beyond this single paper, limited research in humans has been completed. Interestingly, a recent paper did demonstrate a down-regulation of CARNS mRNA in just 5 weeks when subjects were placed on a vegetarian diet (Baguet et al. 2011b), suggesting that BA sarcoplasmic availability has an important impact on CARNS expression. In summary, many of the points of regulation involved in BA delivery and carnosine synthesis appear to have been identified (Fig. 1). However, additional human research is required to fully substantiate all points of regulation and to elucidate the impact that increased BA availability and/or changing carnosine contents could have on each key step of regulation. For more information please refer to Baguet et al. (2012) and Harris et al. (2012) in this carnosine special edition.

\section{Plasma kinetics of $\beta$-alanine consumption in vivo}

Acute oral administration of BA ( $>800 \mathrm{mg}$ ) has consistently been shown to cause transient $(\sim 60-90 \mathrm{~min})$ paraesthesia, 
which has been described, depending on the dose and individual, as mild to severe "pins and needles" and "itching" sensations, which appears linked to plasma BA kinetics (Harris et al. 2006). The mechanism(s) responsible for these paraesthesia symptoms are not yet completely understood, as at least five different BA receptor sites have been identified that could be responsible for sensitization of nociceptive neurons and the associated paraesthesia sideeffects (Crozier et al. 2007; Tiedje et al. 2010). For further information, the interested reader is directed to a primary study published in this special edition by our research group examining the effect of altered BA plasma kinetics, via consumption of slow versus fast release BA tablets, on subsequent paraesthesia symptoms and urinary spillover in humans (Decombaz et al. 2011).

In the first series of studies in humans, it was found that single BA doses greater than $\sim 10 \mathrm{mg} \mathrm{BA} / \mathrm{kg}$ body weight (BW; $\sim 800 \mathrm{mg} / \mathrm{dose}$ ), caused increasing moderate to severe paraesthesia symptoms (Harris et al. 2006). Accordingly, to circumvent undesired paraesthesia, many initial BA dosing studies utilized multiple $800 \mathrm{mg}$ BA doses (sometimes up to eight individual doses) throughout the day to deliver an efficacious daily BA dose for muscle carnosine synthesis (Kendrick et al. 2008, 2009). However, Harris et al. (2006) also examined delivering BA in a concentrated chicken broth, a more complex food matrix that would slow gastric emptying. Interestingly, even at a high dose of $40 \mathrm{mg} / \mathrm{kg} \mathrm{BW}(\sim 3.2 \mathrm{~g}$ of BA), when BA was delivered in $640 \mathrm{ml}$ of chicken broth the peak plasma BA was $50 \%$ lower, with a 50 min later time to peak as compared to a $40 \mathrm{mg} / \mathrm{kg} \mathrm{BW}$ pure BA supplement. Importantly, the former demonstrated no reported paraesthesia symptoms, while in the latter treatment, subjects reported significant symptoms, demonstrating that BA-associated paraesthesia symptoms were related to either/both peak plasma BA concentration and time to peak. Analogous to drug pharmacology, increasingly, more evidences have shown that altered pharmacokinetics of active ingredients in complex food matrices can have considerable impact on in vivo functional outcomes, such as food matrix betaglucan slowing the release of creatine and causing significantly less urinary creatine excretion (Deldicque et al. 2008). As highlighted within this journal, a 'slow-release' BA supplement not only eliminates all paraesthesia symptoms (no measured paraesthesia symptom differences between 'slow-release' BA and placebo) but also results in one-third of the urinary spillover of BA over a $6 \mathrm{~h}$ postsupplementation period (Decombaz et al. 2011). Furthermore, given the serial blood and symptom measurements in this study, a comprehensive BA plasma kinetics to paraesthesia symptoms appraisal was possible. Consequently, it appears that paraesthesia symptoms are more coupled to the rate of plasma $\mathrm{BA}$ increase rather than the peak concentration, as paraesthesia symptoms from pure BA supplementation were greatest at $15 \mathrm{~min}$ with a BA concentration of $82 \mu \mathrm{mol} / \mathrm{L}$, despite the fact that peak values actually reached $\sim 225 \mu \mathrm{mol} / \mathrm{L}$ at $30 \mathrm{~min}$. Despite lowering the plasma peak, these 'slow-release' BA formulations still reach effective plasma concentrations that are above the BA transporter $K_{\mathrm{m}}$ of $\sim 40 \mu \mathrm{M}$ (Bakardjiev and Bauer 1994), as supported by a recent dose-response publication from our laboratory, demonstrating highly efficacious muscle carnosine synthesis with a 'slow-release' supplement (Stellingwerff et al. 2011).

Only a single study has examined whether chronic supplementation of BA with concurrently increasing muscle carnosine concentrations can impact on subsequent paraesthesia (Stellingwerff et al. 2011). Since carnosine requires free histidine during synthesis, it might be hypothesized that prolonged BA supplementation causing increases in carnosine may impact upon L-histidine availability for histamine production and release (Flancbaum et al. 1990), and subsequent histamine-like reactions. However, this study showed no effect of acute or chronic 'slow-release' BA supplementation on paraesthesia symptoms or blood clinical chemistry and hematology measures over the 8-week supplementation period (total BA consumed was $134.4 \mathrm{~g}$; Stellingwerff et al. 2011).

More researches need to be completed to fully characterize the mechanism(s) responsible for the paraesthesia symptoms found in humans with acute high dose BA supplementation. However, paraesthesia can be circumvented with 'slow-release' BA tablets that not only eliminate paraesthesia, but also allow for an easier and more practical BA supplementation protocol, as twice the dose can be acutely consumed ( $\sim 1.6$ vs. $0.8 \mathrm{~g}$ of pure BA) without any significant side-effects. In addition, by eliminating perceived paraesthesia side-effects, 'slow-release' BA supplements allow for optimal blinding of subjects in future performance based studies.

\section{Carnosine synthesis via beta-alanine supplementation}

Despite muscle carnosine having a long scientific history of being associated with improved buffering and performance (Parkhouse et al. 1985; Harris et al. 1990), it was not until 1999 that the hypothesis that muscle carnosine contents could be augmented via oral supplementation of its two constituent amino acids (L-histidine and $\beta$-alanine) was tested by Dunnett and Harris (1999). In this study, they found that $100 \mathrm{mg} / \mathrm{kg} \mathrm{BW} /$ day of $\beta$-alanine with 12.5 $\mathrm{mg} / \mathrm{kg} \mathrm{BW} /$ day of L-histidine supplemented to thoroughbred racehorses (equivalent to $\sim 7 \mathrm{~g} \mathrm{BA}$ /day in humans) over 30 days significantly increased the carnosine content of the 
gluteus medius in five of six tested horses. Their major conclusion from this paper was that "changes in muscle carnosine concentrations appeared to be influenced by beta-alanine bioavailability" (Dunnett and Harris 1999). This conclusion was influenced by the fact that the concentration of L-histidine in muscle and plasma is high compared to the low concentrations of BA $(<2 \mu \mathrm{M}$ plasma $\mathrm{BA}$ ), and transport of BA into the muscle cell is via a highly specific $\beta$-amino-acid transporter with a $K_{\mathrm{m}}$ of $\sim 40 \mu \mathrm{M}$ (Bakardjiev and Bauer 1994). Seven years later, Harris and co-workers (2006) extended these initial findings in thoroughbred racehorses by being the first to show that prolonged BA supplementation ( $\sim 3-6$ g/day over 4 weeks) in humans also causes significant increases in muscle carnosine content $(\sim 40-65 \%)$. Since this seminal paper, every human study thus far has demonstrated significant increases $(>40 \%)$ in muscle carnosine during a chronic BA-supplementation protocol (1.6-6.4 g/day for $>4$ weeks) via either quantification of muscle biopsies (Kendrick et al. 2008, 2009; Harris et al. 2006; Hill et al. 2007) or ${ }^{1} \mathrm{H}-\mathrm{MRS}$, magnetic resonance spectroscopy (MRS) analysis (Baguet et al. 2009, 2010; Derave et al. 2007; Stellingwerff et al. 2011). Thus, the capacity to increase muscle carnosine from BA supplementation in humans appears to be a very robust and reproducible finding. All of these studies are highlighted in Fig. 2, which correlates the percent increase in muscle carnosine versus the total BA consumed at the time of the carnosine quantification.
There is certainly considerable variability between published studies when examining the absolute carnosine concentrations ( $\left.\mathrm{mmol} / \mathrm{kg}_{\mathrm{ww}}\right)$ or the relative (\% change) or absolute increases after BA supplementation (Fig. 2). This variability is due to both physiological and methodological reasons. Probably the number one determinate of carnosine content is the individual fiber type distribution, with Type II 'fast-twitch' fibers containing nearly about double the carnosine content as compared to Type I 'slow-twitch' (Harris et al. 1998), with large differences between individuals (Parkhouse et al. 1985). This in turn could have an impact on the ability of individual fibers to synthesize carnosine, although data thus far appears to show that despite fast-twitch fibers having a greater initial carnosine content, all fibers (Type I or Type IIa; Hill et al. 2007) and muscle groups (Baguet et al. 2009; Stellingwerff et al. 2011) appear equally responsive to carnosine loading via BA supplementation. As reviewed by Derave et al. (2010) other physiological mechanisms that could be responsible for the variability in loading and/or washout may be the age, sex or background dietary intake of BA (e.g., vegetarians) of each individual. Further, it appears that for a given total BA consumed, the percent increase in carnosine is greater in muscle biopsy and biochemical extraction studies than compared to ${ }^{1} \mathrm{H}-\mathrm{MRS}$ methods (Fig. 2). This is not unexpected, as the absolute carnosine values can vary due to measurement location (skeletal muscle type) and methodological differences. An obvious explanation for this apparent discrepancy could be the fact that in all four

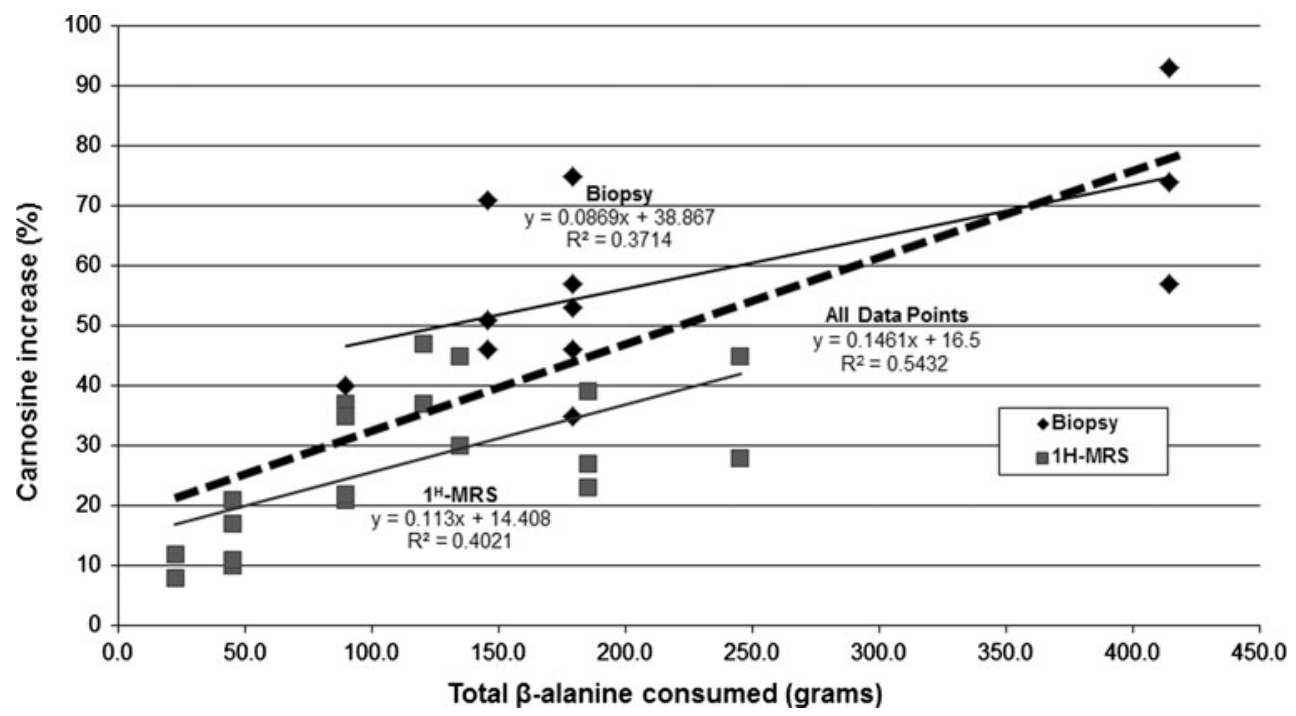

Fig. 2 Correlation of all published studies between the relative percent increases in muscle carnosine as compared to the total $\beta$-alanine consumed by subjects (between 1.6 and $6.4 \mathrm{~g} \mathrm{BA} /$ day). The data represents 31 different muscle carnosine measurements across different durations of BA supplementation (thus different total consumed BA); different muscle groups (e.g., soleus, gastronemius or tibialis anterior via ${ }^{1} \mathrm{H}-\mathrm{MRS}$ ); different muscle fibers (e.g., Type I vs. Type IIa via muscle biopsy and biochemical extraction) and different training states (trained vs. untrained leg). Solid lines compare ${ }^{1} \mathrm{H}$-MRS versus biopsy carnosine quantification, and the dotted line represents all studies together (data adapted from: Kendrick et al. 2008, 2009; Harris et al. 2006; Hill et al. 2007; Baguet et al. 2009, 2010; Derave et al. 2007; Stellingwerff et al. 2011) 
biopsy studies the samples were taken from the m.vastus lateralis (Harris et al. 2006; Kendrick et al. 2008, 2009; Hill et al. 2007), while all the ${ }^{1} \mathrm{H}-\mathrm{MRS}$ data have been obtained from m.tibialis anterior, $\mathrm{m}$-soleus and/or m.gastrocnemius (Stellingwerff et al. 2011; Baguet et al. 2009, 2010; Derave et al. 2007). It has been shown in a recent study (Stellingwerff et al. 2011) that even if the absolute carnosine increases are identical between different muscle groups, the relative increase varies due to the differences in the baseline pre-supplementation levels. Therefore, since biopsy and ${ }^{1} \mathrm{H}$-MRS data are from muscles with different baseline carnosine contents; a systematic difference can be expected. An additional explanation could be that it is becoming increasingly obvious that metabolites can be immobilized (e.g., bound to macromolecules) and are thus not completely MR-visible. Thus, a potential methodological explanation could be limited visibility of all the carnosine metabolites in ${ }^{1} \mathrm{H}-\mathrm{MR}$ spectra, causing a lower carnosine measurement compared to biochemical extraction methods from biopsies, which would quantify all mobile and immobilized metabolites. However, this may not necessarily be a disadvantage for MRS, as the visible metabolites may be the key metabolites involved in biochemical reactions in the cell. Nevertheless, when taking all eight studies together there is a strong positive correlation between the percent increase in muscle carnosine and the total amount of BA consumed. Accordingly, the linear regression (Fig. 2) suggests that for a desired $\sim 50 \%$ in muscle carnosine, a total of $\sim 230 \mathrm{~g}$ of BA must be taken (within a daily consumption range of 1.6-6.4 g BA/ day).

This positive correlation was also strongly demonstrated both in relative (\% increase) and absolute $\left(\mathrm{mmol} / \mathrm{kg}_{\mathrm{ww}}\right.$ increase) terms in a comprehensive dose-response paper that our laboratory recently published (Stellingwerff et al. 2011). The multiple BA doses and multiple carnosine assessments in this study allowed for a univariate assessment which clearly demonstrated a highly linear dependency $\left(R^{2}=0.921\right)$ based on the total grams of $\mathrm{BA}$ consumed, when either 1.6 or $3.2 \mathrm{~g} \mathrm{BA}$ /day was consumed. In this study, it was shown that a total consumption of $100 \mathrm{~g}$ BA increased the absolute carnosine levels by $2.01 \mathrm{mmol} / \mathrm{kg}_{\mathrm{ww}}$. Thus, the absolute $\mathrm{BA} /$ carnosine doseresponse is solely dependent upon the total grams of BA consumed, and there is no impact on the muscle type or the daily BA supplementation regime (within 1.6-6.4 g BA/ day; Fig. 2). These findings result in a greatly simplified BA prescriptive application to augment muscle carnosine. Moreover, the continued apparent linearity (or the nonsaturation) of this correlation suggests that maximal muscle carnosine levels that are potentially attainable remains to be identified via a long-term ( $>6$ months) BA dosing study. It also remains to be seen if it is ideal to recommend absolute daily BA doses (g/day) or correct for lean body mass or body weight $(\mathrm{g} / \mathrm{kg} / \mathrm{day})$. As of yet, no investigation has directly examined the impact that fat free mass (FFM) might have on BA delivery and carnosine synthesis.

Previous data have shown that muscle creatine increases are negatively correlated to high baseline-creatine values (Harris et al. 1992), suggesting a saturation response. This phenomena have helped to explain 'responders' and 'nonresponders', with individuals with low baseline creatine being ideal 'responders' to creatine supplementation and performance outcomes. Thus, it could be hypothesized that individuals with low baseline carnosine [either from a low percentage of Type II fibers (Baguet et al. 2011c; Parkhouse et al. 1985; Harris et al. 1998) or vegetarians, females or the elderly (Everaert et al. 2011)], might respond more favorable to BA-supplemented increases in muscle carnosine. However, contrary to this hypothesis, Baguet et al. (2010) found a positive correlation between high baseline muscle carnosine and the increase in muscle carnosine after supplementation $(n=8)$, meaning the higher the initial carnosine contents the greater the effect of BA supplementation on subsequent carnosine synthesis. However more recently, we found no effect $(p=0.342)$ of baseline carnosine levels and subsequent increases in carnosine via BA supplementation in 21 subjects (Stellingwerff et al. 2011). However, caution should be exercised with both studies as there were a limited total number of subjects, without a huge range of baseline carnosine variability and until it is determined what the maximal attainable muscle carnosine levels are with prolonged BA supplementation (muscle saturation point) correlations between baseline carnosine and increases in carnosine are undoubtedly limited.

Finally, when considering all body tissues containing carnosine that might be impacted by BA supplementation, we have to also consider the brain. Some areas of the human brain are also high in carnosine (e.g., olfactory structures), with homocarnosine being the most abundant type (Kohen et al. 1988), and given the relatively noninvasive nature of ${ }^{1} \mathrm{H}$-MRS theoretically could be quantified. However, as of yet, no human studies have reported whether BA supplementation can also impact upon brain concentrations of carnosine and whether this has an impact on performance and/or health outcomes.

\section{Muscle carnosine washout}

With the cessation of BA supplementation, carnosine degradation and elimination prevails over the synthesis and carnosine washout occurs. However, analogous to carnosine synthesis, the key points of regulation dictating washout of muscle carnosine remain to be clarified. 


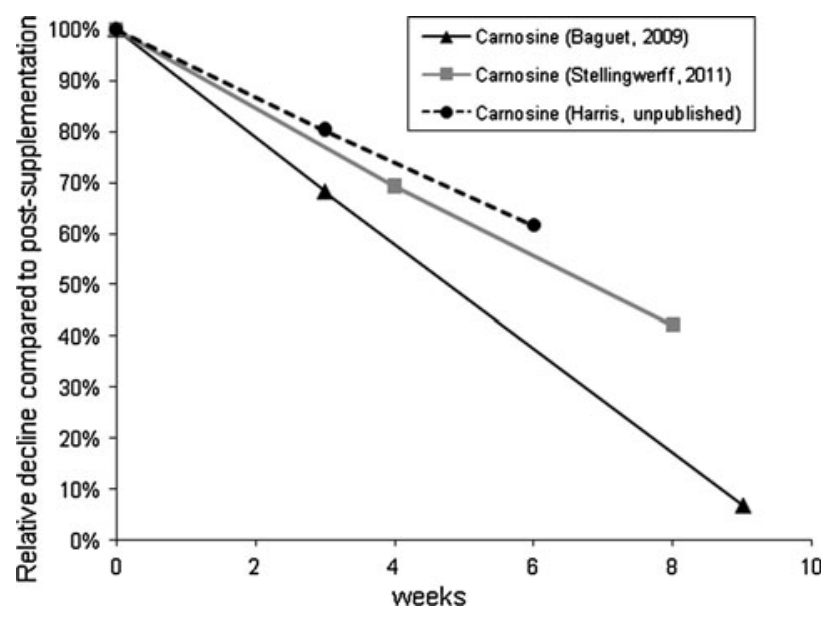

Fig. 3 Relative washout of muscle carnosine in weeks compared to post-supplementation carnosine values (data adapted from: Stellingwerff et al. 2011; Baguet et al. 2009)

Figure 3 is adapted from Baguet et al. (2009) and highlights the two published papers that have examined muscle carnosine washout kinetics post BA-supplementation quantified via ${ }^{1} \mathrm{H}-\mathrm{MRS}$ analysis (Stellingwerff et al. 2011; Baguet et al. 2009), as well as an unpublished study using muscle biopsy and subsequent biochemical quantification of muscle carnosine (Harris, unpublished). Regardless of the carnosine quantification method, the washout of augmented skeletal muscle carnosine after the termination of BA supplementation is very slow, as Fig. 3 shows a washout time of $\sim 10-20$ weeks. This slow washout rate for carnosine is in stark contrast to augmented muscle creatine, which has a washout of $\sim 4$ weeks (Hultman et al. 1996) and is coherent with the net rate of muscle carnosine synthesis being dependent on the total accumulative BA dose, and relatively independent on the supplementation duration (Stellingwerff et al. 2011). Furthermore, carnosine washout appears to be linear in nature and does not feature first-order exponential decay kinetics as found in many enzyme-catalyzed reactions. However, only one of the three studies strictly contradicts an exponential washout (Baguet et al. 2009) while the other two (Harris, unpublished; Stellingwerff et al. 2011) could be explained by either a linear or an exponential washout, given the experimental accuracy. Therefore, further studies are needed to unambiguously support this conclusion.

There appears to be considerable variability for the length of washout time, as Baguet et al. (2009) showed $\sim 10$ weeks for washout, while Stellingwerff et al. (2011) and the unpublished data from Harris demonstrated a $\sim 15$ to 20 -week washout. This variability between studies for calculated washout time is probably due to some of the potential regulatory influences described above regarding carnosine synthesis and degradation and methodological considerations. Furthermore, it is well established that due to continual daily exercise, athletes have increased protein turnover rates (increases in both synthesis and breakdown), and therefore to stay in nitrogen balance athletes require twice the amount of dietary protein intake compared to sedentary individuals (Phillips and Van Loon 2011). Thus, it could be hypothesized that individuals who habitually exercise may have higher carnosine turnover rates which may impact upon synthesis and washout profiles and variability between studies, but needs to be addressed in future work. Nevertheless, given the slow washout time-course of carnosine, any potential performance and/or health benefits resulting from augmented muscle carnosine would appear to be realized 4-6 weeks post-supplementation.

\section{Future directions}

Since 2006, there has been a marked increase of in vivo human studies examining the effects of BA supplementation on muscle carnosine synthesis and the subsequent impact this may have on clinical populations and performance (for reviews see: Sale et al. 2010; Derave et al. 2010; Artioli et al. 2010). However, there are still many questions that remain to completely elucidate the points of regulation of carnosine synthesis, and ultimately, what the optimal BA-dosing protocol and optimal muscle and/or brain carnosine concentration for health and performance are. Important future practical questions and research inquiries are highlighted below:

- What is the peak attainable muscle carnosine, and once peak carnosine levels are realized, will correlations with baseline carnosine become more apparent? What are the performance and health outcomes with ever increasing muscle carnosine contents?

- Could ever increasing muscle carnosine contents ultimately lead to significant decreases in muscle taurine since they both share the same muscle transporter (TauT)?

- Does baseline carnosine contents and subsequent BA dose to carnosine synthesis response and/or peak attainable muscle carnosine differ between different populations and explain some of the variability in responses? (e.g., old/young; disease states; trained/ untrained; male/female; fiber-type differences?)

- What impact do varying baseline carnosine contents have on plasma carnosinase (CN1) activity on subsequent muscle carnosine synthesis via BA supplementation, and vice versa? Does CN1 activity, and other key enzymes and transporters, change with increasing or decreasing muscle carnosine concentrations? 
- What is the minimal dose of BA needed to maintain augmented carnosine stores and could this be achieved with high meat/turkey intake (similar to our ancestors)?

- Are there any interactions with other supplements/ nutrients that impact on delivery, uptake and/or carnosine synthesis (e.g., taurine)?

- Finally, is brain carnosine content also impacted from BA supplementation?

When studying these remaining questions, it is important to consider the need for human in vivo research. More specifically, humans have highly active serum CN1 enzyme activity (Fig. 1; Teufel et al. 2003), in contrast to rodents and some other mammals which lack serum carnosinase, and thus, have considerable amounts of plasma carnosine (Sauerhofer et al. 2007). This, and other fundamental species differences (Abe 2000), will require that nearly all of the mechanistic questions be ascertained via human studies. Taken together, given the large potential for augmented carnosine to positively impact health and sports performance, our quest for knowledge regarding carnosine metabolism must continue to evolve.

Conflict of interest All authors declare that they have no conflict of interest.

\section{References}

Abe H (2000) Role of histidine-related compounds as intracellular proton buffering constituents in vertebrate muscle. Biochemistry (Mosc) 65(7):757-765

Artioli GG, Gualano B, Smith A, Stout J, Lancha AH Jr (2010) Role of beta-alanine supplementation on muscle carnosine and exercise performance. Med Sci Sports Exerc 42(6):1162-1173. doi:10.1249/MSS.0b013e3181c74e38

Baguet A, Reyngoudt H, Pottier A, Everaert I, Callens S, Achten E, Derave W (2009) Carnosine loading and washout in human skeletal muscles. J Appl Physiol 106(3):837-842. doi:10.1152/ japplphysiol.91357.2008

Baguet A, Bourgois J, Vanhee L, Achten E, Derave W (2010) Important role of muscle carnosine in rowing performance. J Appl Physiol 109(4):1096-1101. doi:10.1152/japplphysiol.00141.2010

Baguet A, Everaert I, Achten E, Thomis M, Derave W (2011a) The influence of sex, age and heritability on human skeletal muscle carnosine content. Amino Acids. doi:10.1007/s00726-011-1197-3

Baguet A, Everaert I, De Naeyer H, Reyngoudt H, Stegen S, Beeckman S, Achten E, Vanhee L, Volkaert A, Petrovic M, Taes Y, Derave W (2011b) Effects of sprint training combined with vegetarian or mixed diet on muscle carnosine content and buffering capacity. Eur J Appl Physiol 111(10):2571-2580. doi: 10.1007/s00421-011-1877-4

Baguet A, Everaert I, Hespel P, Petrovic M, Achten E, Derave W (2011c) A new method for non-invasive estimation of human muscle fiber type composition. PLoS ONE 6(7):e21956. doi: 10.1371/journal.pone.0021956

Bakardjiev A, Bauer K (1994) Transport of beta-alanine and biosynthesis of carnosine by skeletal muscle cells in primary culture. Eur J Biochem/FEBS 225(2):617-623
Begum G, Cunliffe A, Leveritt M (2005) Physiological role of carnosine in contracting muscle. Int J Sport Nutr Exerc Metab 15(5):493-514

Bergstrom J, Furst P, Noree LO, Vinnars E (1974) Intracellular free amino acid concentration in human muscle tissue. J Appl Physiol 36(6):693-697

Boldyrev AA, Severin SE (1990) The histidine-containing dipeptides, carnosine and anserine: distribution, properties and biological significance. Adv Enzyme Regul 30:175-194

Crozier RA, Ajit SK, Kaftan EJ, Pausch MH (2007) MrgD activation inhibits $\mathrm{KCNQ} / \mathrm{M}$-currents and contributes to enhanced neuronal excitability. J Neurosci 27(16):4492-4496. doi:10.1523/ JNEUROSCI.4932-06.2007

Decombaz J, Beaumont B, Vuichoud J, Bouisset F, Stellingwerff T (2011) Effect of slow-release b-alanine tablets on absorption kinetics and paresthesia. Amino Acids. doi:10.1007/s00726011-1169-7

del Favero S, Roschel H, Solis MY, Hayashi AP, Artioli GG, Otaduy MC, Benatti FB, Harris RC, Wise JA, Leite CC, Pereira RM, de Sá-Pinto AL, Lancha-Junior AH, Gualano B (2011) Beta-alanine $\left(\right.$ Carnosyn $\left.{ }^{\mathrm{TM}}\right)$ supplementation in elderly subjects (60-80 years): effects on muscle carnosine content and physical capacity. Amino Acids. doi:10.1007/s00726-011-1190-x

Deldicque L, Decombaz J, Foncea HZ, Vuichoud J, Poortmans JR, Francaux M (2008) Kinetics of creatine ingested as a food ingredient. Eur J Appl Physiol 102(2):133-143. doi:10.1007/ s00421-007-0558-9

Derave W, Sale C (2011) Carnosine in Exercise and Disease: an introduction to the International Congress held at the University of Ghent, Belgium, July 2011. Amino Acids: Ghent Carnosine Conference Special Edition update post acceptance

Derave W, Ozdemir MS, Harris RC, Pottier A, Reyngoudt H, Koppo K, Wise JA, Achten E (2007) Beta-alanine supplementation augments muscle carnosine content and attenuates fatigue during repeated isokinetic contraction bouts in trained sprinters. J Appl Physiol 103(5):1736-1743

Derave W, Everaert I, Beeckman S, Baguet A (2010) Muscle carnosine metabolism and beta-alanine supplementation in relation to exercise and training. Sports Med 40(3):247-263. doi: 10.2165/11530310-000000000-00000

Dieck ST, Heuer H, Ehrchen J, Otto C, Bauer K (1999) The peptide transporter PepT2 is expressed in rat brain and mediates the accumulation of the fluorescent dipeptide derivative beta-AlaLys-Nepsilon-AMCA in astrocytes. Glia 25(1):10-20

Drozak J, Veiga-da-Cunha M, Vertommen D, Stroobant V, Van Schaftingen E (2010) Molecular identification of carnosine synthase as ATP-grasp domain-containing protein 1 (ATPGD1). J Biol Chem 285(13):9346-9356. doi:10.1074/jbc.M109.095505

Dunnett M, Harris RC (1999) Influence of oral beta-alanine and Lhistidine supplementation on the carnosine content of the gluteus medius. Equine Vet J Suppl 30:499-504

Everaert I, Mooyaart A, Baguet A, Zutinic A, Baelde H, Achten E, Taes Y, De Heer E, Derave W (2011) Vegetarianism, female gender and increasing age, but not CNDP1 genotype, are associated with reduced muscle carnosine levels in humans. Amino Acids 40(4):1221-1229. doi:10.1007/s00726-010-0749-2

Flancbaum L, Fitzpatrick JC, Brotman DN, Marcoux AM, Kasziba E, Fisher H (1990) The presence and significance of carnosine in histamine-containing tissues of several mammalian species. Agents Actions 31(3-4):190-196

Fritzson P (1957) The catabolism of C14-labeled uracil, dihydrouracil, and beta-ureidopropionic acid in rat liver slices. J Biol Chem 226(1):223-228

Gardner ML, Illingworth KM, Kelleher J, Wood D (1991) Intestinal absorption of the intact peptide carnosine in man, and 
comparison with intestinal permeability to lactulose. J Physiol 439:411-422

Gualano B, Everaert I, Stegen S, Artioli GG, Taes Y, Roschel H, Achten E, Otaduy MC, Lancha-Junior AH, Roger Harris RC, Derave W (2011) Reduced muscle carnosine content in type 2, but not in type 1 diabetic patients. Amino Acids. doi:10.1007/ s00726-011-1165-y

Gulewitsch W, Amiradzibi S (1900) Uber das carnosine, eine neue organische Base des Fleischextraktes. Ber Dtsch Chem Ges 33:1902-1904

Harris RC (2010) Simultaneous changes in muscle carnosine and taurine during and following supplementation with $\beta$-Alanine. In: American College of Sports Medicine, Baltimore, Maryland, USA, vol 5, p 107. doi:10.1249/01.MSS.0000385977.18201.74

Harris RC, Marlin DJ, Dunnett M, Snow DH, Hultman E (1990) Muscle buffering capacity and dipeptide content in the thoroughbred horse, greyhound dog and man. Comp Biochem Physiol A 97(2):249-251

Harris RC, Soderlund K, Hultman E (1992) Elevation of creatine in resting and exercised muscle of normal subjects by creatine supplementation. Clin Sci (Lond) 83(3):367-374

Harris RC, Dunnett M, Greenhaff PL (1998) Carnosine and taurine contents in individual fibres of human vastus lateralis muscle. J Sports Sci 16:639-643

Harris RC, Tallon MJ, Dunnett M, Boobis L, Coakley J, Kim HJ, Fallowfield JL, Hill CA, Sale C, Wise JA (2006) The absorption of orally supplied beta-alanine and its effect on muscle carnosine synthesis in human vastus lateralis. Amino Acids 30(3):279-289

Harris RC, Wise JA, Price KA, Kim HJ, Kim CK, Sale C (2012) Determinants of muscle carnosine content. Amino Acids. doi: 10.1007/s00726-012-1233-y

Hill CA, Harris RC, Kim HJ, Harris BD, Sale C, Boobis LH, Kim CK, Wise JA (2007) Influence of beta-alanine supplementation on skeletal muscle carnosine concentrations and high intensity cycling capacity. Amino Acids 32(2):225-233

Hultman E, Soderlund K, Timmons JA, Cederblad G, Greenhaff PL (1996) Muscle creatine loading in men. J Appl Physiol 81(1): 232-237

Kendrick IP, Harris RC, Kim HJ, Kim CK, Dang VH, Lam TQ, Bui TT, Smith M, Wise JA (2008) The effects of 10 weeks of resistance training combined with beta-alanine supplementation on whole body strength, force production, muscular endurance and body composition. Amino Acids 34(4):547-554

Kendrick IP, Kim HJ, Harris RC, Kim CK, Dang VH, Lam TQ, Bui TT, Wise JA (2009) The effect of 4 weeks beta-alanine supplementation and isokinetic training on carnosine concentrations in type I and II human skeletal muscle fibres. Eur J Appl Physiol 106(1):131-138. doi:10.1007/s00421-009-0998-5
Kohen R, Yamamoto Y, Cundy KC, Ames BN (1988) Antioxidant activity of carnosine, homocarnosine, and anserine present in muscle and brain. Proc Natl Acad Sci USA 85(9):3175-3179

$\mathrm{Lu} \mathrm{H}$, Klaassen C (2006) Tissue distribution and thyroid hormone regulation of Pept1 and Pept 2 mRNA in rodents. Peptides 27(4):850-857. doi:10.1016/j.peptides.2005.08.012

Parkhouse WS, McKenzie DC, Hochachka PW, Ovalle WK (1985) Buffering capacity of deproteinized human vastus lateralis muscle. J Appl Physiol 58(1):14-17

Phillips SM, Van Loon LJ (2011) Dietary protein for athletes: from requirements to optimum adaptation. J Sports Sci 29(Suppl 1):S29-S38. doi:10.1080/02640414.2011.619204

Quinn PJ, Boldyrev AA, Formazuyk VE (1992) Carnosine: its properties, functions and potential therapeutic applications. Mol Aspects Med 13(5):379-444

Sale C, Saunders B, Harris RC (2010) Effect of beta-alanine supplementation on muscle carnosine concentrations and exercise performance. Amino Acids 39(2):321-333. doi:10.1007/ s00726-009-0443-4

Sauerhofer S, Yuan G, Braun GS, Deinzer M, Neumaier M, Gretz N, Floege J, Kriz W, van der Woude F, Moeller MJ (2007) L-carnosine, a substrate of carnosinase-1, influences glucose metabolism. Diabetes 56(10):2425-2432. doi:10.2337/db07-0177

Stellingwerff $\mathrm{T}$, Anwander $\mathrm{H}$, Egger A, Buehler $\mathrm{T}$, Kreis $\mathrm{R}$, Decombaz J, Boesch C (2011) Effect of two beta-alanine dosing protocols on muscle carnosine synthesis and washout. Amino Acids. doi:10.1007/s00726-011-1054-4

Tarnopolsky MA (2011) Creatine as a therapeutic strategy for myopathies. Amino Acids 40(5):1397-1407. doi:10.1007/s00726011-0876-4

Teufel M, Saudek V, Ledig JP, Bernhardt A, Boularand S, Carreau A, Cairns NJ, Carter C, Cowley DJ, Duverger D, Ganzhorn AJ, Guenet C, Heintzelmann B, Laucher V, Sauvage C, Smirnova T (2003) Sequence identification and characterization of human carnosinase and a closely related non-specific dipeptidase. J Biol Chem 278(8):6521-6531. doi:10.1074/jbc.M209764200

Thwaites DT, Anderson CM (2007) $\mathrm{H}^{+}$-coupled nutrient, micronutrient and drug transporters in the mammalian small intestine. Exp Physiol 92(4):603-619. doi:10.1113/expphysiol.2005.029959

Tiedje KE, Stevens K, Barnes S, Weaver DF (2010) Beta-alanine as a small molecule neurotransmitter. Neurochem Int 57(3):177-188. doi:10.1016/j.neuint.2010.06.001

Tomi M, Tajima A, Tachikawa M, Hosoya K (2008) Function of taurine transporter (Slc6a6/TauT) as a GABA transporting protein and its relevance to GABA transport in rat retinal capillary endothelial cells. Biochim Biophys Acta 1778(10): 2138-2142. doi:10.1016/j.bbamem.2008.04.012 